\title{
The PTS transporters of Lactobacillus gasseri ATCC 33323
}

Alyssa L Francl ${ }^{1,3}$, Taksawan Thongaram ${ }^{1}$, Michael J Miller ${ }^{1,2^{*}}$

\begin{abstract}
Background: Lactobacilli can utilize a variety of carbohydrates which reflects the nutrient availability in their respective environments. A common lactobacilli in the human gastrointestinal tract, Lactobacillus gasseri, was selected for further study. The currently available annotation of the L. gasseri ATCC 33323 genome describes numerous putative genes involved in carbohydrate utilization, yet the specific functions of many of these genes remain unknown.

Results: An enzyme I (EI) knockout strain revealed that a functional phosphotransferase transporter system (PTS) is required to ferment at least 15 carbohydrates. Analysis of the L. gasseri ATCC 33323 genome identified fifteen complete (containing all of the necessary subunits) PTS transporters. Transcript expression profiles in response to various carbohydrates (glucose, mannose, fructose, sucrose and cellobiose) were analyzed for the fifteen complete PTS transporters in L. gasseri. PTS 20 was induced 27 fold in the presence of sucrose and PTS 15 was induced 139 fold in the presence of cellobiose. No PTS transporter was induced by glucose, fructose or mannose. Insertional inactivation of PTS 15 and PTS 20 significantly impaired growth on cellobiose and sucrose, respectively. As predicted by bioinformatics, insertional inactivation of PTS 21 confirmed its role in mannose utilization.

Conclusions: The experiments revealed the extensive contribution of PTS transporters to carbohydrate utilization by L. gasseri ATCC 33323 and the general inadequacy of the annotated sugar specificity of lactobacilli PTS transporters.
\end{abstract}

\section{Background}

Lactic Acid Bacteria (LAB) are a group of functionally and genetically related bacteria known for the fermentation of sugars to the metabolic end-product, lactic acid [1]. LAB belong to the order of Lactobacillales, which includes the genera Lactobacillus, Lactococcus, Leuconostoc, Oenococcus, Pediococcus, Streptococcus, among others [2]. LAB, including lactobacilli, are very diverse and are commonly found in many different environments. Lactobacilli are naturally associated with many foods, including fruits, vegetables, cereal grains, wine, milk and meats. In addition, several species of Lactobacillus, such as Lactobacillus gasseri, are considered to be indigenous to the gastrointestinal tract (GIT) and other mucosal surfaces, including the mouth and vagina [3,4]. The Lactobacillus genus has been explored for their probiotic potential due to the ability of specific strains

\footnotetext{
* Correspondence: mille216@illinois.edu

'Department of Food Science and Human Nutrition, University of Illinois, 905
} S. Goodwin Ave., Urbana, IL, USA to survive passage through the human GIT and exert benefits to general health and wellness to the host [5]. Probiotics have been defined as live microorganisms that, when administered in adequate amounts, confer a health benefit to the host [6]. Some of these benefits include a positive influence on the normal microbiota present in the GIT, the competitive exclusion of pathogens, and the stimulation or adjustment of mucosal immunity [7].

Lactobacilli can utilize a variety of carbohydrates which reflects the nutrient availability in their respective environments. In many lactobacilli, PTS (phosphotransferase system) transporters are the dominant carbohydrate transporters [8]. For example, the L. plantarum genome revealed 25 PTS transporters which correlate with its broad carbohydrate utilization profile [9]. Analysis of the L. johnsonii, L. acidophilus and L. gasseri genomes further substantiate these observations since they contain a preponderance of PTS transporters [10]. The PTS functions by the transfer of a phosphate group 
from phosphoenolpyruvate (PEP) to the incoming sugar through a series of sequential steps that involve the different components of the PTS. The PTS consists of cytoplasmic components, which lack sugar specificity, and membrane-associated enzymes, which are specific for a few sugars, at most. The cytoplasmic components are enzyme I $(E I)$ and histidine-phosphorylatable protein (HPr). The membranous component of the PTS system, enzyme II (EII), is made up of three to four subunits: IIA, IIB, IIC and sometimes IID [11].

In reference to the human GIT, lactobacilli are the predominant species in the ileum [12]. The carbohydrate utilization profile of lactobacilli isolated from porcine ileal contents reflects the carbohydrate content of the diet [13]. For example, the relative percentage of lactobacilli that can utilize starch increases after weaning, whereas the relative percentage of lactobacilli that can utilize lactose decreases after weaning. Carbohydrate transporters, including PTS transporters, are among the genes that have been shown to be expressed during GIT transit of lactobacilli $[14,15]$. The importance of PTS transporters in Lactobacillus johnsonii NCC 533 has been verified by studying gut persistence in vivo. Specifically, expression of a PTS transporter annotated as mannose-specific is required for the long-residence phenotype of L. johnsonii NCC 533 [15].

Genome sequencing of selected lactobacilli has enabled researchers to make additional conclusions about the traits and characteristics of these organisms. In 2006, the sequenced genomes of L. gasseri ATCC 33323 and many other lactobacilli were released [16]. The currently available annotation of the L. gasseri ATCC 33323 genome describes numerous genes potentially involved in the uptake and metabolism of carbohydrates, yet the specific functions of these genes remain unknown. Our objective was to characterize PTS transporter functionality in L. gasseri ATCC 33323 using gene knockouts, bioinformatics, comparative carbohydrate utilization assays and transcript expression profiles.

\section{Results and Discussion}

\section{Identification of PTS-Transported Carbohydrates}

As the most common method of carbohydrate utilization in some lactobacilli [17], the PTS transporters in $L$. gasseri ATCC 33323 were selected for further study. PTS transporters require a functional EI to import carbohydrates [18]. Additionally, some non-PTS carbohydrate transporters also require a functional PTS system for full transport activity $[19,20]$. Insertional inactivation of $E I$ in L. gasseri was performed to identify the carbohydrates which require a functional PTS system for utilization (Table 1). L. gasseri ATCC 33323 EI was only able to utilize 2 (D-glucose and D-maltose) of the 17 carbohydrates that the parent strain could utilize, indicating that transporters independent of the PTS system can import these two carbohydrates. The 15 carbohydrates that can be utilized by L. gasseri ATCC 33323 but not by L. gasseri ATCC 33323 EI are D-galactose, D-fructose, D-mannose, $\mathrm{N}$-acetylglucosamine, amygdalin, arbutin, esculin ferric citrate, salicin, D-cellobiose, D-lactose, D-saccharose (sucrose), D-trehalose, amidon (starch), gentiobiose and D-tagatose. These 15 carbohydrates are either (1) imported directly by a PTS transporter and/or (2) imported by a non-PTS carbohydrate transporter that requires a functional PTS system. Examples of non-PTS transporters that require a functional PTS system to import sugars include LacS [19] and RafP [20]. Both LacS and RafP have a PTS IIA-glc domain (PF00358) fused to a permease domain. The PTS IIA-glc domain of these proteins is required for full transport activity. All PTS IIA domains identified in the Conserved Domain Database [21] for L. gasseri ATCC 33323 are a part of PTS transporters. Additionally, $L$. gasseri ATCC 33323 does not have homologs to LacS or RafP. Consequently, we can confirm that (1) L. gasseri ATCC 33323 does not have a LacS or RafP, and (2) $L$. gasseri ATCC 33323 does not have a PTS IIA domain fused to a non-PTS transporter. While it is still possible that there are unknown PTS IIA domains that have not been characterized, we conclude that the majority of these 15 carbohydrates are imported by PTS transporters.

PTS transporters with specificities for many of these carbohydrates (arbutin, amygdalin, salicin, gentiobiose and tagatose) have not been identified amongst lactobacilli. For several of the other carbohydrates, very few PTS transporters have been identified amongst lactobacilli. For example, PTS transporters for D-galactose and D-lactose have only been identified in L. casei $[22,23]$, whereas many other lactobacilli utilize permeases $[24,20]$. Carbohydrates that can be utilized by both L. gasseri ATCC 33323 and L. gasseri ATCC $33323 E I$ (D-glucose and D-maltose) can be transported into the cell by non-PTS mechanism(s). The $L$. gasseri genome encodes two putative permeases with a predicted specificity for glucose [3]. A putative sugar $\mathrm{ABC}$ transporter has also been predicted for maltose [3]. The importance of PTS transporters in L. gasseri ATCC 33323 was revealed based on the carbohydrate utilization profiles of the wild type and $E I$ knockout strains.

\section{PTS Transporters in Lactobacilli}

Bioinformatic analysis was used to characterize the PTS transporters of the sequenced lactobacilli genomes. In total, eleven different species were analyzed, including Lactobacillus acidophilus NCFM, L. brevis ATCC 367, 
Table 1 Carbohydrate utilization profiles of various lactobacilli

\begin{tabular}{|c|c|c|c|c|}
\hline Carbohydrate & L. gasseri ATCC $33323^{a}$ & L. gasseri ATCC 33323 El::MJM75 & L. gasseri $\mathrm{ADH}$ & L. gasseri ATCC 19992 \\
\hline D-galactose & + & - & + & + \\
\hline D-glucose & + & + & + & + \\
\hline D-fructose & + & - & + & + \\
\hline D-mannose & + & - & + & + \\
\hline $\mathrm{N}$-acetylglucosamine & + & - & + & + \\
\hline Amygdalin & + & - & - & - \\
\hline Arbutin & + & - & - & - \\
\hline Esculin ferric citrate & + & - & + & + \\
\hline Salicin & + & - & - & - \\
\hline D-cellobiose & + & - & + & + \\
\hline D-maltose & + & + & + & + \\
\hline D-lactose (bovine origin) & + & - & + & + \\
\hline D-saccharose (sucrose) & + & - & + & + \\
\hline D-trehalose & + & - & + & + \\
\hline Amidon (starch) & + & - & + & - \\
\hline Gentiobiose & + & - & + & + \\
\hline D-tagatose & + & - & + & + \\
\hline
\end{tabular}

The carbohydrate utilization profiles of L. gasseri ATCC 33323, L. gasseri ATCC 33323 El::MJM75, L. gasseri ADH and L. gasseri ATCC 19992 were determined using API $50 \mathrm{CH}$ assays after 48 hours incubation. The ability or inability to utilize carbohydrates is represented by " + " or "-", respectively. The superscript indicates the following: a - there were no differences among the carbohydrate utilization profiles of L. gasseri ATCC 33323 PTS 15::MJM99, L. gasseri ATCC 33323 PTS 20:: MJM100, L. gasseri ATCC 33323 PTS 21::MJM101 and L. gasseri ATCC 33323.

L. casei ATCC 334, L. delbrueckii ssp. bulgaricus ATCC 11842, L. delbrueckii ssp. bulgaricus ATCC BAA-365, L. gasseri ATCC 33323, L. johnsonii NCC 533, L. plantarum WCFS1, L. reuteri F275, L. sakei ssp. sakei $23 \mathrm{~K}$ and L. salivarius ssp. salivarius UCC118. A complete PTS transporter was defined as having the IIA, IIB and IIC components present in the enzyme II of the PTS. An incomplete PTS lacked one or more of these three subunits [25].

Table 2 lists the eleven different lactobacilli and the number of complete and incomplete PTS(s) found in

Table 2 Complete and incomplete PTS transporters in selected lactobacilli

\begin{tabular}{lcc}
\hline \multicolumn{1}{c}{ Organism } & $\begin{array}{c}\text { Complete } \\
\text { PTS }\end{array}$ & $\begin{array}{c}\text { Incomplete } \\
\text { PTS }\end{array}$ \\
\hline L. acidophilus NCFM & 10 & 13 \\
L. brevis ATCC 367 & 0 & 5 \\
L. casei ATCC 334 & 17 & 14 \\
L. delbrueckii ssp. bulgaricus ATCC 11842 & 2 & 7 \\
L. delbrueckii ssp. bulgaricus ATCC BAA-365 & 2 & 4 \\
L. gasseri ATCC 33323 & 15 & 10 \\
L. johnsonii NCC 533 & 16 & 9 \\
L. plantarum WCFS1 & 25 & 13 \\
L. reuteri F275 & 0 & 4 \\
L. sakei ssp. sakei 23 K & 5 & 6 \\
L. salivarius ssp. Salivarius UCC118 & 7 & 3 \\
\hline Complete transorts we deined as having the & IIA and IIC subunits
\end{tabular}

Complete transporters were defined as having the IIA, IIB and IIC subunits of Ell present, and incomplete transporters were defined as lacking at least one subunit. each organism. The number of PTS transporters in the selected lactobacilli analyzed varies greatly. L. plantarum WCFS1 has the most complete PTS transporters with 25, whereas L. reuteri F275 and L. brevis ATCC 367 have no complete PTS transporters. The closely related L. gasseri ATCC 33323, L. johnsonii NCC 533 and L. acidophilus NCFM had 15, 16 and 10 complete PTS transporters, respectively.

The number of PTS transporters present in a species has been proposed to be due to the adaptation of species to their specific niches [26]. Species such as L. gasseri ATCC 33323, L. acidophilus NCFM and $L$. johnsonii NCC 533 all have more PTS transporters than most of the other species. These common inhabitants of the GIT may require a large number of PTS transporters to survive in their environment. L. delbrueckii species are commonly used in dairy fermentations, where the nutrient-rich environment has less carbohydrate diversity and has resulted in significant gene loss in respect to carbohydrate utilization [27].

In an effort to characterize PTS transporters through bioinformatics, seven different PTS families have been differentiated [25] and are available at the Transport Classification Database [28]. Table 3 lists the PTS transporter families for all of the complete and incomplete PTS transporters in L. gasseri ATCC 33323. Two of the three complete PTS transporters from the LAC family (PTS 6 and 9) have no known homologs amongst the 10 other lactobacilli analyzed (listed in Table 2). In addition, PTS 8, of which none of the other 10 analyzed 
Table 3 Current annotations and predicted substrates of the PTS transporters in L. gasseri ATCC 33323

\begin{tabular}{|c|c|c|c|c|}
\hline PTS & ORF & Current annotation & Predicted Function & TCDB Family [40] \\
\hline $1 \mathrm{~B}$ & 117 & $\begin{array}{l}\text { PTS, mannose/fructose/N-acetylgalactosamine-specific } \\
\text { component IIB }\end{array}$ & & 4.A.6 \\
\hline $1 \mathrm{C}$ & 118 & $\begin{array}{l}\text { PTS, mannose/fructose/N-acetylgalactosamine-specific } \\
\text { component IIC }\end{array}$ & & \\
\hline $1 \mathrm{D}$ & 119 & $\begin{array}{l}\text { PTS, mannose/fructose/N-acetylgalactosamine-specific } \\
\text { component IID }\end{array}$ & & \\
\hline $1 \mathrm{~A}$ & 120 & PTS, mannose/fructose-specific component IIA & & \\
\hline $2 \mathrm{~A}$ & 125 & $\begin{array}{l}\text { Phosphotransferase system galacitol-specific IIA domain } \\
\text { (Ntr-type) }\end{array}$ & & 4.A.6 \\
\hline $3 B C A$ & 149 & PTS fructose-specific component IIB & & 4.A.2 \\
\hline $4 C$ & 187 & Cellobiose-specific PTS system IIC component & & 4.A.3 \\
\hline $5 \mathrm{~A}$ & 192 & Cellobiose-specific PTS system IIA component & & 4.A.3 \\
\hline $5 B$ & 194 & Cellobiose-specific PTS system IIB component & & \\
\hline $5 C$ & 195 & Cellobiose-specific PTS system IIC component & & \\
\hline $6 \mathrm{~A}$ & 342 & Cellobiose-specific PTS system IIA component & Lactose $^{b_{1}, c, d} ;$ Galactose $^{c}$ & 4.A.3 \\
\hline $6 C B$ & 343 & Cellobiose-specific PTS system IIC component & & \\
\hline 7BCA & 398 & Sucrose PTS, EIIBCA & & 4.A.1 \\
\hline $8 \mathrm{~A}$ & 495 & PTS, galacitol-specific IIA domain (Ntr-type) & Lactose $^{c}$; Galactose ${ }^{c}$ & 4.A.5 \\
\hline $8 \mathrm{~B}$ & 496 & PTS, galacitol-specific IIB component & & \\
\hline $8 \mathrm{C}$ & 497 & Galactitol PTS, EllC & & \\
\hline $9 \mathrm{~A}$ & 500 & Cellobiose-specific PTS system IIA component & & 4.A.3 \\
\hline $9 \mathrm{CB}$ & 501 & Cellobiose-specific PTS system IIC component & & \\
\hline $10 \mathrm{~B}$ & 514 & $\begin{array}{l}\text { PTS, mannose/fructose/N-acetylgalactosamine-specific } \\
\text { component IIB }\end{array}$ & Galactose $^{c}$ & 4.A.6 \\
\hline $10 \mathrm{C}$ & 515 & $\begin{array}{l}\text { PTS, mannose/fructose/N-acetylgalactosamine-specific } \\
\text { component IIC }\end{array}$ & & \\
\hline $10 \mathrm{D}$ & 516 & $\begin{array}{l}\text { PTS, mannose/fructose/N-acetylgalactosamine-specific } \\
\text { component IID }\end{array}$ & & \\
\hline $10 \mathrm{~A}$ & 517 & PTS, mannose/fructose-specific component IIA & & \\
\hline $11 \mathrm{ABC}$ & 535 & Beta-glucoside-specific PTS system IIABC component & Trehalose $^{a}$ & 4.A.1 \\
\hline $12 \mathrm{C}$ & 570 & Cellobiose-specific PTS system IIC component & & 4.A.3 \\
\hline $13 \mathrm{~A}$ & 1348 & Glucitol/sorbitol PTS, EllA & & - \\
\hline $14 \mathrm{C}$ & 1430 & Cellobiose-specific PTS system IIC component & & 4.A.3 \\
\hline $15 B C A$ & 1669 & Trehalose PTS trehalose component IIBC & Cellobiose $^{c, d} ; \beta$-glucosides ${ }^{a}$; Galactose $^{c}$ & 4.A.1 \\
\hline $16 C$ & 1676 & Cellobiose-specific PTS system IIC component & & 4.A.3 \\
\hline $17 \mathrm{CBA}$ & 1688 & $\mathrm{~N}$-acetylglucosamine and glucose PTS, EllCBA & & 4.A.1 \\
\hline $18 \mathrm{ABC}$ & 1726 & $\begin{array}{l}\text { Fusion of }\|\mathrm{A},\| \mathrm{IB} \text { and } \| \mathrm{C} \text { component of } \\
\text { mannitol/fructose-specific PTS }\end{array}$ & Fructose $^{b}$ & 4.A.2 \\
\hline $19 B C A$ & 1755 & Beta-glucosides PTS, EIIBCA & & 4.A.1 \\
\hline $20 B C A$ & 1778 & Sucrose PTS, EIIBCA & Sucrose ${ }^{b, c, d}$ & 4.A.1 \\
\hline $21 \mathrm{D}$ & 1793 & Mannose-specific PTS system component IID & Glucose $^{a}$; Mannose $e^{a, d}$ & 4.A.6 \\
\hline $21 C$ & 1794 & Mannose-specific PTS system component IIC & & \\
\hline $21 \mathrm{AB}$ & 1795 & PTS, mannose/fructose-specific component IIAB & & \\
\hline $22 \mathrm{C}$ & 1811 & Cellobiose-specific PTS system IIC component & & 4.A.3 \\
\hline $23 \mathrm{C}$ & 1835 & Galacitol PTS, EIIC & & 4.A.5 \\
\hline $24 \mathrm{C}$ & 1836 & Galacitol PTS, EllC & & 4.A.5 \\
\hline $25 \mathrm{C}$ & 1851 & Cellobiose-specific PTS system IIC component & & 4.A.3 \\
\hline
\end{tabular}

The superscripts for the predicted functions indicate the following: $a$ - homology to characterized PTS transporters in other species; $b$ - homology to PTS transporters that are induced by a particular carbohydrate(s) in other species; c - PTS transporters that are induced by a particular carbohydrate in L. gasseri ATCC 33323; and d - characterization in L. gasseri ATCC 33323. The TCDB family names are categorized as follows: $4 . A .1$ - PTS glucose-glucoside (GLC); 4.A.2 PTS fructose-mannitol (FRU); $4 . A .3$ - PTS Lactose-N,N'-Diacetylchitobiose- $\beta$-glucoside (LAC); $4 . A .5$ - PTS Galactitol (GAT); and 4.A.6 - PTS Mannose-FructoseSorbose (MAN) [40]. 
lactobacilli have a complete homolog, is the only complete PTS member of the GAT family in L. gasseri ATCC 33323. There are no members of the GUT and ASC family amongst the 15 complete PTS transporters of L. gasseri ATCC 33323.

\section{Strain Variation}

In order to determine the variability of PTS transporters within $L$. gasseri, fifteen complete PTS transporters in $L$. gasseri ATCC 33323 were compared to L. gasseri ADH and L. gasseri ATCC 19992 using PCR (Table 4). PCR products were obtained for all of the fifteen PTS transporters when L. gasseri ATCC 33323 was used as the template. There was no visible amplicon for PTS 6 and 9 for either L. gasseri ADH or ATCC 19992. In addition, there was no visible amplicon for PTS 7 and 10 in L. gasseri ADH. The PCR of all other PTS transporters resulted in a visible product for L. gasseri ADH and L. gasseri ATCC 19992. The PTS transporters that are unique to $L$. gasseri ATCC 33323 amongst sequenced lactobacilli (PTS 6, 7 and 9) also appear to be variable within L. gasseri.

Recently, draft genomic DNA sequences have become publicly available from three L. gasseri strains (202-4, MV-22 and JV-V03). Bioinformatic analysis of the L. gasseri draft genomes revealed that PTS 7, 10 and 15 from L. gasseri ATCC 33323 are not present in all L. gasseri strains whereas the other 12 complete PTS transporters in L. gasseri ATCC 33323 where also found in L. gasseri 202-4, L. gasseri MV-22 and L. gasseri JV-V03. While caution should be used to interpret the draft genomes since they are unfinished, it is interesting to note that

Table 4 Presence of complete L. gasseri ATCC 33323 PTS transporters in other $L$. gasseri strains

\begin{tabular}{|c|c|c|c|}
\hline $\begin{array}{l}\text { L. gasseri ATCC } \\
33323 \text { PTS }\end{array}$ & $\begin{array}{c}\text { L. gasseri ATCC } \\
33323\end{array}$ & $\begin{array}{l}\text { L. gasseri } \\
\mathrm{ADH}\end{array}$ & $\begin{array}{c}\text { L. gasseri ATCC } \\
19992\end{array}$ \\
\hline 1 & + & + & + \\
\hline 3 & + & + & + \\
\hline 5 & + & + & + \\
\hline 6 & + & - & - \\
\hline 7 & + & - & + \\
\hline 8 & + & + & + \\
\hline 9 & + & - & - \\
\hline 10 & + & - & + \\
\hline 11 & + & + & + \\
\hline 15 & + & + & + \\
\hline 17 & + & + & + \\
\hline 18 & + & + & + \\
\hline 19 & + & + & + \\
\hline 20 & + & + & + \\
\hline 21 & + & + & + \\
\hline
\end{tabular}

The presence or absence of a visible PCR gel product in $L$. gasseri ATCC 33323, L. gasseri ADH and L. gasseri ATCC 19992 is denoted by "+" or "-", respectively.
PTS 7 and PTS 10 were found to be variable amongst $L$. gasseri using both PCR and bioinformatic approaches.

Carbohydrate utilization assays were also used to study different $L$. gasseri strains in comparison to L. gasseri ATCC 33323. L. gasseri ADH and L. gasseri ATCC 19992 had different carbohydrate utilization profiles when compared to L. gasseri ATCC 33323, as shown in Table 1. Among the L. gasseri strains, only L. gasseri ATCC 33323 was able to grow on amygdalin, arbutin and salicin. Both L. gasseri ATCC 33323 and L. gasseri ADH were able to grow on amidon (starch), but L. gasseri ATCC 19992 was not able to grow on amidon. Also, there were no carbohydrates that were unique to L. gasseri ATCC 19992. As previously indicated [29], these results demonstrate the potential for gain/loss of carbohydrate utilization genes which results in difficulty in using carbohydrate utilization assays for species identification.

\section{Transcript Expression Profiles}

Real-time PCR was used to study the transcript expression profiles of the fifteen complete PTS transporters in L. gasseri ATCC 33323 in response to fructose (calibrator), glucose, mannose, cellobiose and sucrose. PTS 7 and PTS 20 were annotated as being sucrose-specific and both have adjacent ORFs annotated at sucrose-6phosphate hyrdolase. PTS 20 was induced $27 \pm 19$ fold with sucrose as the sole carbohydrate source, whereas all other PTS transporters were induced less than 3 fold (Figure 1A). The L. acidophilus NCFM PTS transporter (ORF 401) induced by sucrose [24] is a homolog of PTS 20 (80\% amino acid identity). In fact, L. johnsonii NCC 533 ORF 519 is also a homolog to PTS 20 in L. gasseri (98\% amino acid identity), and all three strains can utilize sucrose.

In the presence of cellobiose, PTS 15 was induced 139 \pm 97 fold (Figure 1B). All other PTS transporters were induced less than 5 fold. L. acidophilus NCFM has a homolog to PTS 15 (ORF 725 at 62\% amino acid identity) and is able to utilize cellobiose. Surprisingly, three of the complete PTS transporters of L. gasseri ATCC 33323 were annotated as cellobiose-specific (PTS 5, 6 and 9), yet none demonstrated inducible expression in the presence of cellobiose. The annotation of PTS 15 incorrectly indicates a specificity for trehalose, yet PTS 11 is a homolog for the characterized trehalose PTS in L. acidophilus NCFM [30]. Our results demonstrate the importance of determining PTS transcript expression profiles to identify PTS transporter specificity rather than relying solely on annotation and bioinformatics.

There were no PTS transporters that were significantly induced in the presence of glucose or mannose (Figures $1 \mathrm{C}$ and 1D, respectively). The PTS transporter for glucose is constitutively expressed in Streptococcus mutans [31], S. bovis [32], and Lactobacillus casei [33]. 
A

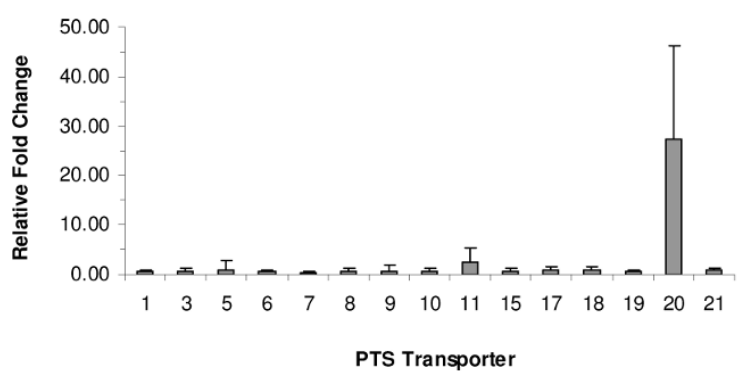

C

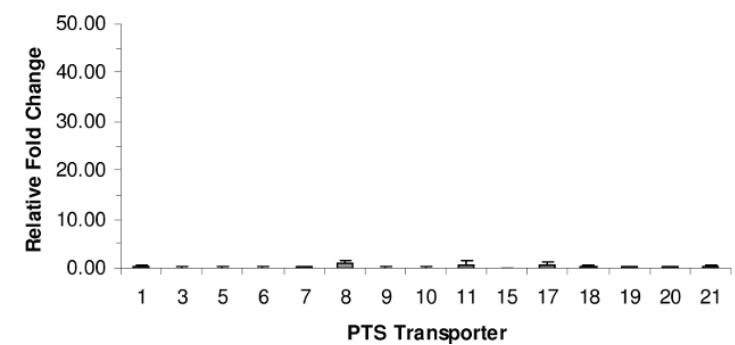

B

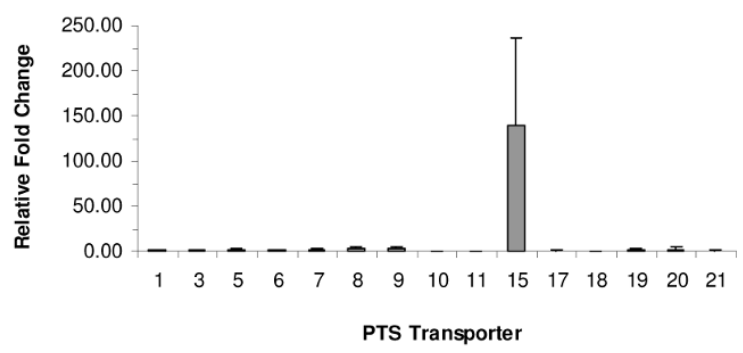

D

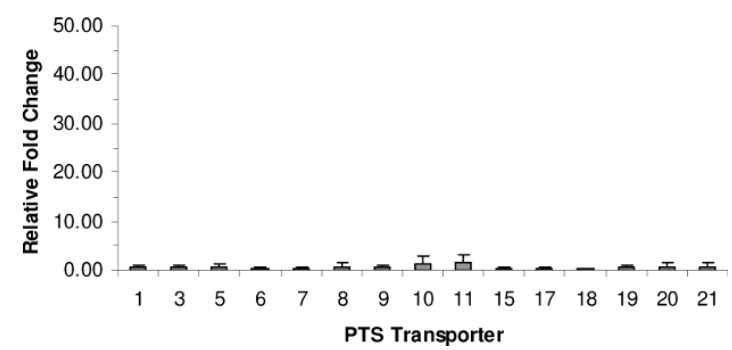

Figure 1 Relative fold changes of the complete PTS transporters in L. gasseri ATCC 33323. Cells grown in semi-synthetic MRS + selected carbohydrate were compared to cells grown in semi-synthetic MRS + fructose. Selected carbohydrates were sucrose (A), cellobiose (B), glucose (C) and mannose (D). RNA was extracted from log phase cells and subjected to two-step real-time PCR. Results are the average of three independent experiments, and error bars indicate standard deviations.

Additionally, no PTS transporter was induced by glucose in L. acidophilus NCFM [24]. PTS 21 includes a fused IIA and IIB domain (ORF 1795), in addition to the enzyme IID (ORF 1793) subunit which are characteristic of glucose PTS transporters [34]. In addition, PTS 21 is a homolog of the characterized glucose/mannose PTS transporter in L. casei [33], providing evidence that PTS 21 may be involved in the transport of glucose. Homologs of PTS 21 are found in all 8 of the sequenced lactobacilli genomes we analyzed that contain at least one complete PTS transporter. L. gasseri ATCC 33323 EI indicates that a non-PTS mechanism is able to import glucose as well (Table 1). While no PTS transporter was induced by mannose (Figure 1D), PTS transporter function is required for the utilization of mannose (Table 1), suggesting that the glucose permease(s) is unable to transport mannose. Since the glucose PTS transporter can also transport mannose in some instances [31], and that the PTS 21 homolog in L. casei ATCC 393 transports glucose and mannose [33,35], we predict that PTS 21 also transports glucose and mannose.

\section{Insertional Inactivation of PTS 15, PTS 20 and PTS 21}

In order to confirm the conclusions from bioinformatic and transcript analyses, gene knockouts for PTS 15 (MJM99), PTS 20 (MJM100) and PTS 21 (MJM101) were created. Carbohydrate utilization assays were used to characterize MJM99, MJM100 and MJM101 (Table 1). No differences were detected among these three knockout strains and the parental strain. The qualitative nature of the carbohydrate utilization assay prevented the ability to characterize these knockout strains.

Growth curves were performed with MJM99, MJM100, MJM101, L. gasseri ATCC 33323 (NCK334) and L. gasseri ATCC 33323 EI (MJM75) (Figure 2). The growth media had sucrose (Figure 2A), cellobiose (Figure 2B), glucose (Figure $2 \mathrm{C}$ ) or mannose (Figure $2 \mathrm{D}$ ) as the sole carbohydrate. In all four cases, L. gasseri ATCC 33323 EI did not grow and was indistinguishable from the noninoculated control. Growth of MJM100 was significantly reduced on sucrose (Figure 2A), confirming the bioinformatic and transcript expression profile based prediction. Growth of MJM99 was significantly reduced on cellobiose (Figure 2B), confirming the transcript expression profile based prediction. In regards to glucose, the growth of all four knockout strains was similar to the parental strain (Figure 2C). MJM101 had a significantly extended lag phase that was approximately 10 hours longer than the lag phase observed with the other analyzed strains when mannose was the sole carbohydrate (Figure 2D). PTS 21 and another unidentified PTS transporter(s) import mannose. 


\section{Prediction of L. gasseri ATCC 33323 PTS Transporter Specificities}

We have identified 15 carbohydrates that require a functional PTS system for utilization (Table 1): galactose, fructose, mannose, $\mathrm{N}$-acetylglucosamine, amygdalin, arbutin, esculin ferric citrate, salicin, cellobiose, lactose, sucrose, trehalose, starch, gentiobiose and tagatose. The annotations of the complete and incomplete PTS transporters are presented in Table 3. Sucrose induced expression of PTS 20 (Figure 1A), and cellobiose induced expression of PTS 15 (Figure 1B). Insertional inactivation of PTS 20 and PTS 15 significantly reduced growth on sucrose (Figure 2B) and cellobiose (Figure $2 \mathrm{C}$ ), respectively. Based on transcription expression profiles, bioinformatics and the characterization of a PTS 21 knockout strain, we predict that PTS 21 can transport glucose and mannose [33].

We could not detect increased PTS expression from any of the 15 complete PTS transporters when grown in fructose with the glucose condition as the calibrator. However, PTS 3 and PTS 18 are two candidates for fructose transport. Both PTS 3 and PTS 18 co-localize with ORFs (LGAS_0148 and LGAS_1727, respectively) which have a fructose-1-phosphate kinase domain
(FruK; COG 1105). PTS 18 is a homolog to the PTS transporter in L. acidophilus (LBA1777) which is induced in the presence of fructose [24], yet we were unable to demonstrate induction of PTS 18 or any other complete PTS transporter with fructose. PTS 3 does not have a homolog in L. acidophilus NCFM. Additionally, PTS 3 and/or PTS 18 may be involved in tagatose utilization. The potential activity of COG 1105 includes tagatose-6-phosphate kinase which is required for the tagatose-6-phosphate pathway. Unfortunately, no PTS transporter amongst LAB has been demonstrated to transport tagatose. However, L. acidophilus NCFM is unable to utilize tagatose and also lacks a homolog for PTS 3. Functional characterization is required to determine if PTS 3 and/or PTS 18 transports fructose and/or tagatose.

Previous studies have identified a lactose permease in the closely related $L$. acidophilus NCFM [24]. However, L. gasseri ATCC 33323 does not have a homolog for the lactose permease from $L$. acidophilus NCFM. Rather, $L$. gasseri ATCC 33323 uses PTS transporters to import lactose. PTS 6 and PTS 8 are induced by lactose [36]. Analysis of L. gasseri PTS 6, L. gasseri PTS 8 and L. gasseri PTS 6 PTS 8 revealed that PTS 6 is required for

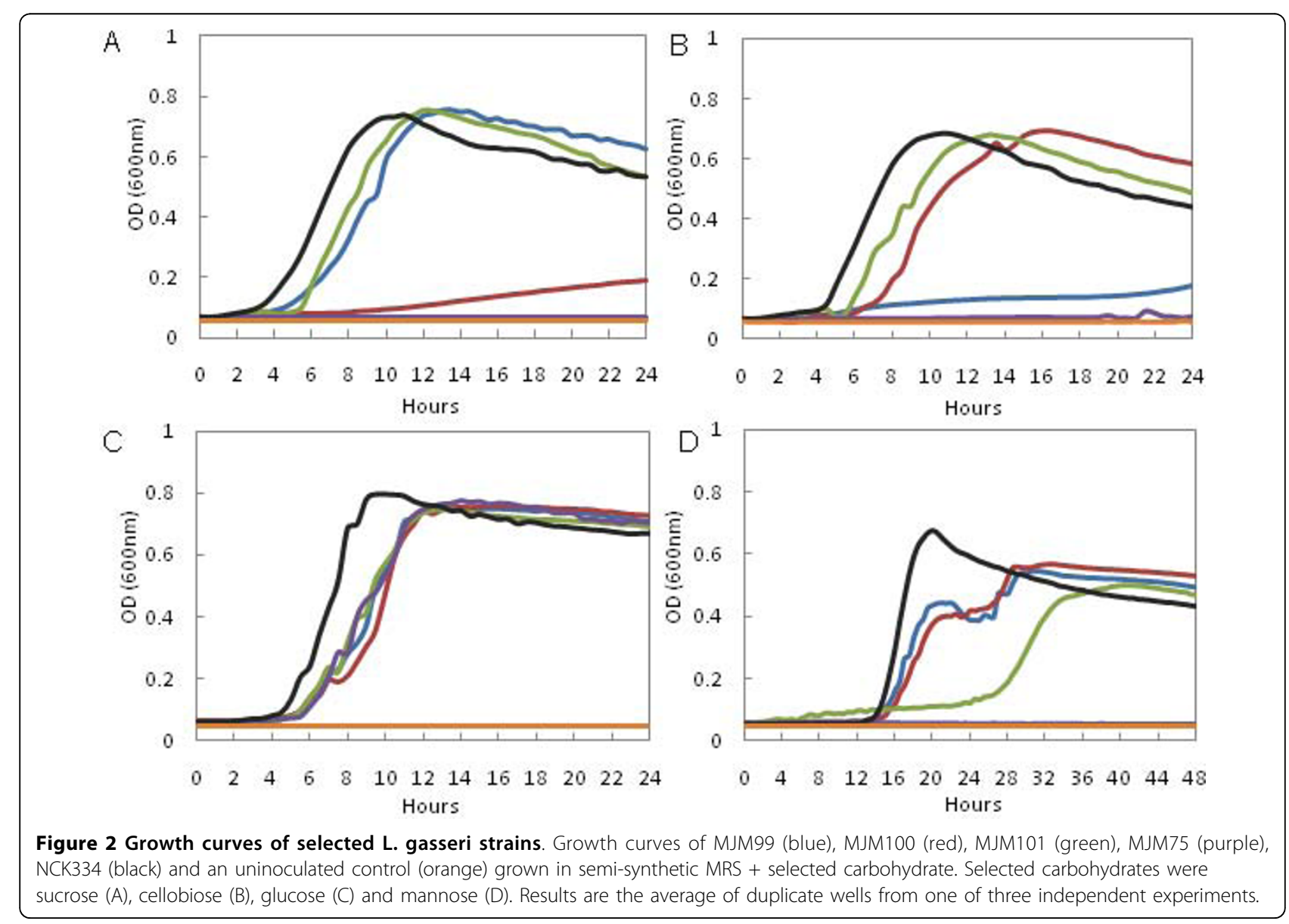


maximum fermentation of lactose [36]. The only lactose PTS transporter previously characterized in lactobacilli has been with $L$. casei $[22,23]$. Galactose induced several PTS transporters (PTS 6, 8, 10 and 15) [36]. Similar to lactose, analysis of L. gasseri PTS 6, L. gasseri PTS 8 and L. gasseri PTS 6 PTS 8 revealed that PTS 6 is required for maximum fermentation of galactose [36].

PTS 11 is a homolog for the PTS transporter in $L$. acidophilus (ORF 1012) which is induced in the presence of trehalose and is required for the utilization of trehalose [30]. In addition, LGAS_0533 is homologous to the phosphotrehalase (treC) characterized in L. acidophilus NCFM. While PTS 11 has an $\alpha$-glucosidase near (treC), no predicted $\beta$-glucosidase is in the PTS 11 operon, suggesting that PTS 11 may not involved in $\beta$ glucoside uptake as annotated.

No PTS transporter that transports N-acetylglucosamine has been characterized in LAB. Based on our current knowledge, we can not predict which PTS transporter(s) can import $\mathrm{N}$-acetylglucosamine.

We have identified several $\beta$-glucosides that are likely imported by PTS transporters including arbutin, salicin, gentiobiose, amygdalin and cellobiose. PTS 15 is the major cellobiose PTS transporter in L. gasseri ATCC 33323. Cellobiose PTS transporters have been identified that also transport other $\beta$-glucosides $[37,38]$. In addition, PTS 15 is a homolog to a PTS transporter in Streptococcus mutans that transports $\beta$-glucoside esculin [39]. PTS 5, 6, 8, 9, 15 and 19 all co-localize with potential $\beta$ glucosidases. Additional characterization is needed to identify which PTS transporters are involved in the utilization of $\beta$-glucosides.

\section{Conclusions}

PTS transporters were confirmed to be largely important in the carbohydrate utilization potential of L. gasseri ATCC 33323. The PTS transporters were identified in various lactobacilli species using bioinformatic analysis. Comparative carbohydrate utilization assays were used to analyze the PTS content with carbohydrate utilization capability of three L. gasseri strains. The PTS carbohydrate specificity of transporters in L. gasseri ATCC 33323 was characterized by studying the transcript expression profiles in response to different carbohydrates. Lastly, the growth activity of selected PTS knockouts confirmed PTS transporter specificity predictions based on bioinformatics and transcript expression profiles. Our results confirm the importance of combining bioinformatics, transcript expression profiles and gene inactivation in identifying carbohydrate specificity of PTS transporters.

\section{Methods}

\section{Bioinformatic Analysis}

The genomes of Lactobacillus acidophilus NCFM, $L$. brevis ATCC 367, L. casei ATCC 334, L. delbrueckii ssp. bulgaricus ATCC 11842, L. delbrueckii ssp. bulgaricus ATCC BAA-365, L. gasseri ATCC 33323, L. johnsonii NCC 533, L. plantarum WCFS1, L. reuteri F275, L. sakei ssp. sakei $23 \mathrm{~K}$ and L. salivarius ssp. salivarius UCC118 were analyzed using Concise Protein BLAST [40]. The PTS transporters of these strains were compared based on sequence similarity and function. PTS transporters were placed in the same cluster based on reciprocal best-hit blastP scores. Homologs were defined as PTS transporters that were in the same cluster.

The number of complete and incomplete PTS transporters present was determined for each species through bioinformatic analysis of the genomes. A complete PTS transporter was defined as having complete EIIA, EIIB and EIIC domains, which are required for PTS functionality [25]. An incomplete PTS transporter (also known as an orphan PTS) was defined as lacking in at least one of these domains. The sequential numbering of PTS transporters was based on their location in each respective genome. In order to identify non-PTS transporters with a PTS IIA domain, the conserved domain database was searched for PTS IIA domains [21,41].

\section{Bacterial Strains, Plasmids and Growth Conditions}

The bacterial strains and plasmids used in this study are listed in Table 5. L. gasseri strains were grown at $37^{\circ} \mathrm{C}$, in deMan, Rogosa, Sharpe (MRS) broth (Difco, Sparks, MD) or on MRS supplemented with 1.5\% agar (Fisher, Fair Lawn, NJ). Agar plates were incubated anaerobically in a Coy anaerobic chamber (Grass Lake, MI) with a gas composition of $90 \%$ nitrogen, $5 \%$ hydrogen and $5 \%$ carbon dioxide. When necessary, erythromycin (Fisher) was added at a concentration of $2.5 \mu \mathrm{g} / \mathrm{mL}$, and chloramphenicol (Fisher) was added at a concentration of $5 \mu \mathrm{g} / \mathrm{mL}$. For the real-time PCR studies, L. gasseri ATCC 33323 was grown at $37^{\circ} \mathrm{C}$ in $10 \mathrm{~mL}$ semi-synthetic MRS medium supplemented with $1 \%$ carbohydrate (wt/vol) as previously described [42], except bromocresol purple was not used.

Escherichia coli cells were grown at $37^{\circ} \mathrm{C}$, in Luria-Bertani (LB) broth (Fisher) or on LB supplemented with $1.5 \%$ agar and grown anaerobically. When appropriate, kanamycin (Teknova, Hollister, CA) was added at a concentration of $40 \mu \mathrm{g} / \mathrm{mL}$, erythromycin (Fisher) was added at a concentration of $150 \mu \mathrm{g} / \mathrm{mL}$, and chloramphenicol (Fisher) was added at a concentration of $15 \mu \mathrm{g} / \mathrm{mL}$. 


\section{DNA Isolation, Manipulations and Transformations}

Genomic DNA was isolated from $L$. gasseri ATCC 33323 using the Microbial DNA Isolation kit (MO BIO, Carlsbad, CA) according to the manufacturer's protocol. E. coli plasmid DNA was isolated using the QIAprep Spin Miniprep kit (QIAGEN).

DNA manipulations were carried out according to standard procedures. Restriction enzymes and T4 ligase were obtained from Invitrogen (Carlsbad, CA). When necessary, DNA fragments were isolated from agarose gels using the Zymoclean Gel DNA Recovery kit (Zymo Research, Orange, CA). PCR reactions were carried out according to standard procedures using EconoTaq polymerase from Lucigen (Middleton, WI). PCR primers were designed using Clone Manager 9 (Sci-Ed Software, Raleigh, NC) and purchased from IDT (Coralville, IA). For cloning purposes, restriction enzyme sites were added at the 5' end of the primers. PCR products were purified using the DNA Clean and Concentrator kit (Zymo Research).

Electrocompetent L. gasseri ATCC 33323 cells were prepared using $3.5 \times$ sucrose $\mathrm{MgCl}$ electroporation buffer as previously described [43]. To perform the electroporation, the Electroporator 2510 (Eppendorf, Westbury, $\mathrm{NY}$ ) was used at a setting of $2.5 \mathrm{kV}$.

\section{PTS Content of $L$. gasseri Strains}

Additional L. gasseri strains were analyzed for the presence of the fifteen complete PTS transporters found in L. gasseri ATCC 33323 using PCR. Genomic DNA isolated from L. gasseri 33323, L. gasseri ADH and L. gasseri ATCC 19992 was used as templates in the PCR reactions. The same primers used for transcript analysis were used to determine the presence of homologous gene fragments (Table 6).

\section{El Gene Inactivation}

The inactivation of $E I$ was performed by targeted insertion of an erythromycin resistant, non-replicative vector pMJM-1 by homologous recombination using a previously established method [44]. Plasmid pMJM-1 was designed to disrupt the L. gasseri ATCC 33323 EI gene, encoding for enzyme I of the PTS system. The primers AF_1360Bam and AF_1360Nco (Table 6) were used to amplify an $836 \mathrm{bp}$ internal region of $E I$ from L. gasseri. This fragment was cloned via the $\mathrm{BamHI} / \mathrm{Ncol}$ sites into pORI28, an $\mathrm{Ori}^{+}$, RepA integration plasmid. Plasmid pMJM-1 was introduced into L. gasseri containing pTRK669 (MJM79) by electroporation. RepA function was provided by the helper plasmid pTRK669, which is stable at $37^{\circ} \mathrm{C}$ but not at $43^{\circ} \mathrm{C}$. Transformants carrying

Table 5 Bacterial strains and plasmids

\begin{tabular}{|c|c|c|}
\hline Strain or plasmid & Relevant characteristics & Source or reference \\
\hline \multicolumn{3}{|l|}{ L. gasseri } \\
\hline NCK334 & ATCC 33323 , human intestinal isolate & ATCC \\
\hline MJM79 & ATCC 33323 with pTRK669 & This study \\
\hline MJM75 & ATCC 33323 El::pMJM-1, El ${ }^{-}$ & This study \\
\hline MJM99 & ATCC 33323 PTS 15::pMJM-4, PTS $15^{-}$ & This study \\
\hline MJM100 & ATCC 33323 PTS 20::pMJM-5, PTS 20- & This study \\
\hline MJM101 & ATCC 33323 PTS 21::pMJM-6, PTS 21- & This study \\
\hline NCK100 & $\mathrm{ADH}$, human intestinal isolate & [43] \\
\hline MJM55 & ATCC 19992 & ATCC \\
\hline \multicolumn{3}{|l|}{ E. coli } \\
\hline EC 1000 & $\begin{array}{l}\text { RepA }{ }^{+} \text {MC1000, } \mathrm{Km}^{\mathrm{r}} \text {, carrying a single copy of the pWV1 repA gene in the glgB gene; } \\
\text { host for pORI28-based plasmids }\end{array}$ & [44] \\
\hline NCK1609 & EC1000(pORI28) & [44] \\
\hline NCK1391 & EC1000(pTRK669) & [44] \\
\hline MJM80 & EC1000(pMJM-1) & This study \\
\hline MJM103 & EC1000(pMJM-4) & This study \\
\hline MJM104 & EC1000(pMJM-5) & This study \\
\hline MJM105 & EC1000(pMJM-6) & This study \\
\hline \multicolumn{3}{|l|}{ Plasmids } \\
\hline pORI28 & $\mathrm{Em}^{\mathrm{r}}$, ori (pWV1), replicates only with repA provided in trans & [44] \\
\hline pTRK669 & ori (pWV01), $\mathrm{Cm}^{\mathrm{r}}$, provides repA in trans, temperature sensitive & [44] \\
\hline pMJM-1 & $2.5 \mathrm{~kb}, \mathrm{pORI} 28$ with 836-bp internal L. gasseri ATCC 33323 El fragment & This study \\
\hline pMJM-4 & 2.5 kb, pORI28 with 819-bp internal L. gasseri ATCC 33323 PTS 15 fragment & This study \\
\hline pMJM-5 & 2.4 kb, pORI28 with 760-bp internal L. gasseri ATCC 33323 PTS 20 fragment & This study \\
\hline pMJM-6 & $2.3 \mathrm{~kb}$, pORI28 with 675-bp internal L. gasseri ATCC 33323 PTS 21 fragment & This study \\
\hline
\end{tabular}


Table 6 Primer sequences used for transcript expression profiles and gene inactivation

\begin{tabular}{|c|c|c|c|}
\hline Amplified Region & ORF & Primer Sequence & Product Size $(\mathrm{bp})$ \\
\hline \multirow[t]{2}{*}{ PFK } & 881 & 5'-GTTATGGGTCGTGATGTG-3' (F) & 85 \\
\hline & & 5'-AAGGCTCTTCTGGGATAAC-3' (R) & \\
\hline \multirow[t]{2}{*}{ PTS 1} & 118 & 5'-TTGGACGTGGCTTAGTTC-3' (F) & 85 \\
\hline & & 5'-GCACCAGCTACTGTTAAACC-3' (R) & \\
\hline \multirow[t]{2}{*}{ PTS 3} & 149 & 5'-GATCAGGGCTAGTTGTTG-3' (F) & 96 \\
\hline & & 5'-CGGCAGCTAAATAACCAC-3' (R) & \\
\hline \multirow[t]{2}{*}{ PTS 5} & 195 & 5'-GAAGCCTGCGTAAATAAGC-3' (F) & 100 \\
\hline & & 5'-GTTGCCTGAACAAGTTCC-3' (R) & \\
\hline \multirow[t]{2}{*}{ PTS 6} & 343 & 5'-CGCAAATGGATACCATGAAAG-3' (F) & 77 \\
\hline & & 5'-TCCAGTAGTGGTAATCATACG-3' (R) & \\
\hline \multirow[t]{2}{*}{ PTS 7} & 398 & 5'-GGAATGATGGGAAAGGGAATAG-3' (F) & 99 \\
\hline & & 5'-TGCATGACCTGTTGGATAAG-3' (R) & \\
\hline \multirow[t]{2}{*}{ PTS 8} & 497 & 5'-AGCAACCCTATGACTACTACTC-3' (F) & 92 \\
\hline & & 5'-AGCCATGGTAAGCACTTATC-3' (R) & \\
\hline \multirow[t]{2}{*}{ PTS 9} & 501 & 5'-CAACTTGTGCGAAGAATTTAAC-3' (F) & 95 \\
\hline & & 5'-AATTTCAGCAGCTAAGATAACG-3' (R) & \\
\hline \multirow[t]{2}{*}{ PTS 10} & 515 & 5'-GCTCCAGCTTATGTCGTTAG-3' (F) & 109 \\
\hline & & 5'-AGAAGCACCAGTTCGAATAG-3' (R) & \\
\hline \multirow[t]{2}{*}{ PTS 11} & 535 & 5'-GCCAGCCGTTTATGGTATC-3' (F) & 82 \\
\hline & & 5'-AACAAGGCCACCAAATGC-3' (R) & \\
\hline \multirow[t]{2}{*}{ PTS 15} & 1669 & 5'-CTTCATTCCGATAGCATGTC-3' (F) & 100 \\
\hline & & 5'-TGAAGGAGTTGCTGTTGAG-3' (R) & \\
\hline \multirow[t]{2}{*}{ PTS 17} & 1688 & 5'-TCCAGGTGTCTTGAAAGTAG-3' (F) & 99 \\
\hline & & 5'-TCAGGGTGAGTGATAATGTC-3' (R) & \\
\hline \multirow[t]{2}{*}{ PTS 18} & 1726 & 5'-CCATACCACCTACCATAAACAG-3' (F) & 91 \\
\hline & & 5'-GCTTACGTCTTTGCTTCAG-3' (R) & \\
\hline \multirow[t]{2}{*}{ PTS 19} & 1755 & 5'-CAATCATTGCACCATACATAGG-3' (F) & 78 \\
\hline & & 5'-ATTAAGGCGGAATTACAGAAC-3' (R) & \\
\hline \multirow[t]{2}{*}{ PTS 20} & 1778 & 5'-GCGCTTACCGTATACAAAGG-3' (F) & 89 \\
\hline & & 5'-TGGTGCCAAAGAGTATTCC-3' (R) & \\
\hline \multirow[t]{2}{*}{ PTS 21} & 1794 & 5'-GCAGGAATGGCAAGTAATAAAC-3' (F) & 86 \\
\hline & & 5'-GCTATTGATCGTTGGCAAATG-3' (R) & \\
\hline AF_1360Bam & 1360 & 5'-ATGCGGATCC-CGGCAGCCATAGTATATTG-3' (F) & 836 \\
\hline AF_1360Nco & & 5'-ATGCCCATGG-TCATGGCTCGTTCATTAG-3' (R) & \\
\hline AF_ori+ & - & 5'-GATAATGAACTGTGCTGATTAC-3' (F) & 1071 \\
\hline AF_El+ & & 5'-TGGGTTATATGGTTGGTAAAG-3' (R) & \\
\hline AF_ori- & - & 5'-TTCAATCGCCAACGAATC-3' (F) & 1020 \\
\hline AF_El- & & 5'-AGTGATACAGCTCAACTTAAC-3' (R) & \\
\hline MM_1669Bam & 1669 & 5'-AGTCGGATCCTGCAGCAGGTATGATTAAAG-3' (F) & 819 \\
\hline MM_1669Nco & & 5'-AGTCCCATGGAATAGCTGGTTCAGTAACAC-3' (R) & \\
\hline AF_ori+ & - & 5'-GATAATGAACTGTGCTGATTAC-3' (F) & 999 \\
\hline MM_PTS15+ & & 5'-TGCTGATGACGATTTAGATG-3' (R) & \\
\hline AF ori- & - & 5'-TTCAATCGCCAACGAATC-3' (F) & 1039 \\
\hline MM_PTS15- & & 5'-GCTGCAATACAACTTAAGAC-3' (R) & \\
\hline MM_1778Bam & 1778 & 5'-AGTCGGATCCCAGGTTTGTTTGGAGCAAAG' (F) & 760 \\
\hline MM_1778Nco & & 5'-AGTCCCATGGTGCTGGTTCAGTAATACCAAG-3' (R) & \\
\hline AF_ori+ & - & 5'-GATAATGAACTGTGCTGATTAC-3' (F) & 894 \\
\hline MM_PTS20+ & & 5'-CTTAGTAGCTGGTGGTTTG-3' (R) & \\
\hline
\end{tabular}


Table 6: Primer sequences used for transcript expression profiles and gene inactivation (Continued)

\begin{tabular}{clll}
\hline AF ori- & - & 5'-TTCAATCGCCAACGAATC-3' (F) $^{\prime}$ & 990 \\
MM_PTS20- & & 5'-TACACCTGCACCAATTAAAG-3' (R) & 675 \\
\hline MM_1795Bam & 1795 & 5'-AGTCGGATCCAAGGTCCTGATGATATTAGAG' (F) & \\
MM_1795Nco & & 5'-AGTCCCATGGATAGCTTTAAGCGCATCTTC-3' (R) & 854 \\
\hline AF_ori+ & - & 5'-GATAATGAACTGTGCTGATTAC-3' (F) & \\
MM_PTS21+ & & 5'-TAGTCACGGTGGCTTTG-3' (R) & 895 \\
AF ori- & - & 5'-TTCAATCGCCAACGAATC-3' (F) & \\
MM_PTS21- & & 5'-CACTGTAAGCCATGGAAC-3' (R) & \\
\hline
\end{tabular}

both plasmids were transferred five times (overnight transfers) and allowed to grow at $43^{\circ} \mathrm{C}$ in MRS broth supplemented with erythromycin $(2.5 \mu \mathrm{g} / \mathrm{mL})$ to avoid the insertion of multiple copies of the vector.

The occurrence of single cross-over events was verified by PCR amplification of the junction fragments from chromosomal DNA of $\mathrm{Em}^{\mathrm{r}}-\mathrm{Cm}^{\mathrm{s}}$ colonies. EI specific external primers and specific internal primers for the Em gene in the vector were used to confirm successful insertion of pMJM-1 into the EI gene. The 5' junction fragment, demonstrating integration in the $E I$ gene (the primers AF_ori+ and AF_EI+ were used - Table 6) had an expected size of $1071 \mathrm{bp}$. The 3' junction fragment, demonstrating integration in the $E I$ gene (the primers of AF_ori- and AF_EI- were used - Table 6) had an expected size 1020 bp. MJM75 had the expected junction fragments and is an $E I$ knockout.

\section{PTS 15, 20 and 21 Gene Inactivation}

The inactivation of PTS 15, 20 and 21 followed the same general outline as the $E I$ gene inactivation. The non-replicative vectors pMJM-4, pMJM-5 and pMJM-6 were used to inactivate PTS 15, 20, and 21, respectively (Table 5). The amplified PTS 15 (LGAS_1669), 20 (LGAS_1778) and 21 (LGAS_1795) internal regions were 819 bp, 760 bp and 675 bp, respectively. The junction fragments for successful pMJM-4 integration were $999 \mathrm{bp}$ and $1039 \mathrm{bp}$. The junction fragments for successful pMJM-5 integration were $894 \mathrm{bp}$ and $990 \mathrm{bp}$. The junction fragments for successful pMJM-6 integration were 854 bp and 895 bp. MJM99, MJM100 and MJM101 had the expected junction fragments and are PTS 15, PTS 20 and PTS 21 knockouts, respectively.

\section{Carbohydrate Utilization Analysis}

Strains were analyzed for their ability to utilize carbohydrates with the API 50 carbohydrate utilization assay (bioMérieux, Durham, NC) according to the manufacturer's protocol. Strains analyzed are as follows: L. gasseri ATCC 33323, L. gasseri ATCC 33323 EI::MJM75, L. gasseri ADH, L. gasseri ATCC 19992, L. gasseri ATCC
33323 PTS 15::MJM99, L. gasseri ATCC 33323 PTS 20:: MJM100, and L. gasseri ATCC 33323 PTS21::MJM101.

For the growth experiments, L. gasseri strains were first grown in MRS. After two passes, the strains were inoculated into semi-synthetic MRS medium supplemented with $1 \%$ carbohydrate (wt/vol). The growth curve was generated using the protocol described by Barboza et al. [45]. Briefly, $100 \mu \mathrm{l}$ of inoculated media was placed into a sterile 96-well plate and then topped with $40 \mu \mathrm{L}$ of mineral oil. The plate was incubated at $37^{\circ} \mathrm{C}$ in an anaerobic chamber with $\mathrm{OD}_{600 \mathrm{~nm}}$ readings taken every 30 minutes.

\section{RNA Isolation and Analysis}

RNA was isolated from L. gasseri ATCC 33323 using the Microbial RNA Isolation kit (MO BIO) according to the manufacturer's protocol. Semi-synthetic MRS was used to analyze PTS gene expression in response to various carbohydrates. The carbohydrates added to the medium were glucose (Fisher), mannose (Acros Organics, NJ), fructose (Sigma-Aldrich, St. Louis, MO), sucrose (Fisher), or cellobiose (Acros Organics). 0.1\% of overnight culture was transferred 6 times before isolation of RNA. The final transfer of L. gasseri was grown to an $\mathrm{OD}_{595} \mathrm{~nm}$ of 0.6 in order to obtain mid-log phase cells [42]. $1.5 \mathrm{~mL}$ of culture was collected by centrifugation at $10,000 \times g$ at room temperature. RNA was isolated from the cells using the UltraClean Microbial RNA Isolation Kit according to manufacturer's protocol (MO BIO). To eliminate contaminating DNA, $100 \mathrm{ng} /$ $\mu \mathrm{L}$ of RNA was treated with TURBO DNA-free according to the supplier's instructions in a $50 \mu \mathrm{L}$ reaction volume (Ambion, Austin, TX).

Two-step real-time PCR was performed to carry out the relative quantification of the fifteen complete PTS transporters from the five different conditions (glucose, mannose, fructose, sucrose and cellobiose). The reverse transcription step was performed using the iScript cDNA sythesis kit to convert the RNA samples to cDNA according to the manufacturer's protocol (BioRad, Hercules, CA). Typically, $0.8 \mu \mathrm{g}$ of RNA was 
converted to cDNA in a $20 \mu \mathrm{L}$ reaction volume. The iScript PCR reaction conditions used are as follows. The reaction mixture was held at $25^{\circ} \mathrm{C}$ for 5 minutes, $42^{\circ} \mathrm{C}$ for 30 minutes, heated to $85^{\circ} \mathrm{C}$ for 5 minutes, and stored at $4^{\circ} \mathrm{C}$ (Biorad, Hercules, CA). The quantification step of real-time PCR was performed using iTaq SYBR Green Mastermix with ROX (Biorad). Primers were designed for the 15 complete PTS transporters in L. gasseri ATCC 33323 using Clone Manager 9 (Sci-Ed Software) and are shown in Table 6 . The IIC component of each of the fifteen complete PTS transporters was targeted for primer design. Primers used in the real-time experiments were synthesized by Invitrogen.

Relative quantification of the transcription profiles of the fifteen complete PTS transporters in L. gasseri ATCC 33323 was performed using the 7300 Real-time PCR System (Applied Biosystems, Foster City, CA). Typically, $5 \mu \mathrm{L}$ of cDNA $(0.8 \mu \mathrm{g})$ was added to the reaction mixture consisting of $12.5 \mu \mathrm{L}$ iTaq SYBR Green Mastermix with ROX (BioRad), $1 \mu \mathrm{L}$ of the forward primer $(5 \mu \mathrm{M}), 1 \mu \mathrm{L}$ of the reverse primer $(5 \mu \mathrm{M})$, and 5.5 $\mu \mathrm{L}$ DEPC water (MO BIO). The reaction mixture was held at $95^{\circ} \mathrm{C}$ for 2 minutes, $95^{\circ} \mathrm{C}$ for 15 seconds and $60^{\circ}$ $\mathrm{C}$ for one minute (repeated 35 times), $95^{\circ} \mathrm{C}$ for $15 \mathrm{sec}$ onds, $60^{\circ} \mathrm{C}$ for 1 minute, $95^{\circ} \mathrm{C}$ for 15 seconds, and $60^{\circ} \mathrm{C}$ for 15 seconds. Relative fold changes were reported by using a phosphofructokinase (PFK) gene in L. gasseri (Table 6 - PFK primer sequences) that was previously shown in L. plantarum WCFS1 to exhibit qualities of an acceptable internal standard [46]. The ${ }^{\Delta \Delta} \mathrm{Ct}$ method [47] was used to calculate the relative fold change of the PTS systems using fructose as the calibrator. Reported relative fold changes are the average of three independent experiments $+/$ - the standard deviation.

\section{Acknowledgements}

We acknowledge Rodolphe Barrangou and Tri Duong for insightful discussions and technical help. This project was supported by the USDA Cooperative State Research, Education and Extension Service, Hatch project number \# ILLU-698-339. Alyssa Francl was supported by the Bill and Agnes Brown Fellowship. The authors would also like to acknowledge Julia Willett for her help in bioinformatic analysis.

\section{Author details}

'Department of Food Science and Human Nutrition, University of Illinois, 905 S. Goodwin Ave., Urbana, IL, USA. ${ }^{2}$ Division of Nutritional Sciences, University of Illinois, 905 S. Goodwin Ave., Urbana, IL, USA. ${ }^{3}$ Danisco, USA, Inc., 3329 Agriculture Drive, Madison, WI 53716, USA.

\section{Authors' contributions}

ALF performed the majority of the experiments, participated in bioinformatic analysis, study design, and in crafting of the manuscript. TT performed the growth experiments. MJM created MJM99, MJM100, and MJM101, conceived the study, participated in the design, coordination, bioinformatic analysis, and crafting of the manuscript.

Received: 31 August 2009

Accepted: 12 March 2010 Published: 12 March 2010

\section{References}

1. Kandler O: Carbohydrate metabolism in lactic acid bacteria. Antonie van Leeuwenhoek 1983, 49(3):209.

2. Hutkins RW: Microbiology and Technology of Fermented Foods Chicago, III.; Ames, lowa: IFT Press; Blackwell Pub, 12006.

3. Azcarate-Peril MA, Altermann E, Goh YJ, Tallon R, Sanozky-Dawes RB, Pfeiler EA, O'Flaherty S, Buck BL, Dobson A, Duong T, Miller MJ, Barrangou R, Klaenhammer TR: Analysis of the genome sequence of Lactobacillus gasseri ATCC 33323 reveals the molecular basis of an autochthonous intestinal organism. Appl Environ Microbiol 2008, 74(15):4610.

4. Reuter G: The Lactobacillus and Bifidobacterium microflora of the human intestine: composition and succession. Curr Issues Intest Microbiol 2001 2(2):43.

5. Liévin-Le Moal $V$, Servin AL: The front line of enteric host defense against unwelcome intrusion of harmful microorganisms: mucins, antimicrobial peptides, and microbiota. Clin Microbiol Rev 2006, 19(2):315.

6. Reid G, Sanders ME, Gaskins HR, Gibson GR, Mercenier A, Rastall R, Roberfroid M, Rowland I, Cherbut C, Klaenhammer TR: New scientific paradigms for probiotics and prebiotics. J Clin Gastroenterol 2003, 37(2):105.

7. Ouwehand AC, Salminen S, Isolauri E: Probiotics: an overview of beneficial effects. Antonie van Leeuwenhoek 2002, 82(1-4):279.

8. Lorca GL, Barabote RD, Zlotopolski V, Tran C, Winnen B, Hvorup RN, Stonestrom AJ, Nguyen E, Huang LW, Kim DS, Saier MH Jr: Transport capabilities of eleven gram-positive bacteria: comparative genomic analyses. Biochim Biophys Acta 2007, 1768(6):1342.

9. Kleerebezem M, Boekhorst J, van Kranenburg R, Molenaar D, Kuipers OP, Leer R, Tarchini R, Peters SA, Sandbrink HM, Fiers MW, Stiekema W, Lankhorst RM, Bron PA, Hoffer SM, Groot MN, Kerkhoven R, de Vries M, Ursing B, de Vos WM, Siezen RJ: Complete genome sequence of Lactobacillus plantarum WCFS1. Proc Natl Acad Sci USA 2003, 100(4):1990.

10. Klaenhammer TR, Barrangou R, Buck BL, Azcarate-Peril MA, Altermann E: Genomic features of lactic acid bacteria effecting bioprocessing and health. FEMS Microbiol Rev 2005, 29(3):393.

11. Reizer J, Saier MH Jr: Modular multidomain phosphoryl transfer proteins of bacteria. Curr Opin Struct Biol 1997, 7(3):407.

12. Hao WL, Lee YK: Microflora of the gastrointestinal tract: a review. Methods Mol Biol 2004, 268:491

13. Pieper $R$, Janczyk P, Zeyner A, Smidt H, Guiard V, Souffrant WB: Ecophysiology of the developing total bacterial and lactobacillus communities in the terminal small intestine of weaning piglets. Microb Ecol 2008, 56(3):474.

14. Bron PA, Grangette C, Mercenier A, de Vos WM, Kleerebezem M: Identification of Lactobacillus plantarum genes that are induced in the gastrointestinal tract of mice. J Bacteriol 2004, 186(17):5721.

15. Denou E, Pridmore RD, Berger B, Panoff JM, Arigoni F, Brüssow H: Identification of genes associated with the long-gut-persistence phenotype of the probiotic Lactobacillus johnsonii strain NCC533 using a combination of genomics and transcriptome analysis. J Bacteriol 2008, 190(9):3161.

16. Makarova K, Slesarev A, Wolf Y, Sorokin A, Mirkin B, Koonin E, Pavlov A, Pavlova N, Karamychev V, Polouchine N, Shakhova V, Grigoriev I, Lou Y, Rohksar D, Lucas S, Huang K, Goodstein DM, Hawkins T, Plengvidhya V, Welker D, Hughes J, Goh Y, Benson A, Baldwin K, Lee JH, Díaz-Muñiz I, Dosti B, Smeianov V, Wechter W, Barabote R, Lorca G, Altermann E, Barrangou R, Ganesan B, Xie Y, Rawsthorne H, Tamir D, Parker C, Breidt F, Broadbent J, Hutkins R, O'Sullivan D, Steele J, Unlu G, Saier M, Klaenhammer T, Richardson P, Kozyavkin S, Weimer B, Mills D: Comparative genomics of the lactic acid bacteria. Proc Natl Acad Sci USA 2006, 103(42):15611.

17. Postma PW, Lengeler JW, Jacobson GR: Phosphoenolpyruvate: carbohydrate phosphotransferase systems of bacteria. Microbiol Rev 1993, 57(3):543.

18. Cvitkovitch DG, Boyd DA, Thevenot T, Hamilton IR: Glucose transport by a mutant of Streptococcus mutans unable to accumulate sugars via the phosphoenolpyruvate phosphotransferase system. J Bacteriol 1995, 177(9):2251

19. Gunnewijk MGW, Poolman B: HPr(His P)-mediated phosphorylation differently affects counterflow and proton motive force-driven uptake 
via the lactose transport protein of Streptococcus thermophilus. J Biol Chem 2000, 275(44):34080.

20. Leong-Morgenthaler P, Zwahlen MC, Hottinger H: Lactose metabolism in Lactobacillus bulgaricus: analysis of the primary structure and expression of the genes involved. J Bacterio/ 1991, 173(6):1951.

21. The Conserved Domain Database. http://www.ncbi.nlm.nih.gov/cdd.

22. Alpert CA, Chassy BM: Molecular cloning and nucleotide sequence of the factor Illlac gene of Lactobacillus casei. Gene 1988, 62(2):277.

23. Alpert CA, Chassy BM: Molecular cloning and DNA sequence of lacE, the gene encoding the lactose-specific enzyme II of the phosphotransferase system of Lactobacillus casei. Evidence that a cysteine residue is essential for sugar phosphorylation. J Biol Chem 1990, 265(36):22561.

24. Barrangou R, Azcarate-Peril MA, Duong T, Conners SB, Kelly RM, Klaenhammer TR: Global analysis of carbohydrate utilization by Lactobacillus acidophilus using CDNA microarrays. Proc Natl Acad Sci USA 2006, 103(10):3816.

25. Barabote RD, Saier MH Jr: Comparative genomic analyses of the bacterial phosphotransferase system. Microbiol Mol Biol Rev 2005, 69(4):608.

26. Pfeiler EA, Klaenhammer TR: The genomics of lactic acid bacteria. Trends Microbiol 2007, 15(12):546.

27. Guchte van de M, Penaud S, Grimaldi C, Barbe V, Bryson K, Nicolas P, Robert C, Oztas S, Mangenot S, Couloux A, Loux V, Deryyn R, Bossy R, Bolotin A, Batto JM, Walunas T, Gibrat JF, Bessières P, Weissenbach J, Ehrlich SD, Maguin E: The complete genome sequence of Lactobacillus bulgaricus reveals extensive and ongoing reductive evolution. Proc Natl Acad Sci USA 2006, 103(24):9274.

28. TCDB: Transport Classification Database. http://www.tcdb.org/.

29. Berger B, Pridmore RD, Barretto C, Delmas-Julien F, Schreiber K, Arigoni F, Brüssow $\mathrm{H}$ : Similarity and differences in the Lactobacillus acidophilus group identified by polyphasic analysis and comparative genomics. $J$ Bacteriol 2007, 189(4):1311.

30. Duong T, Barrangou R, Russell WM, Klaenhammer TR: Characterization of the tre locus and analysis of trehalose cryoprotection in Lactobacillus acidophilus NCFM. Appl Environ Microbiol 2006, 72(2):1218.

31. Liberman ES, Bleiweis AS: Transport of glucose and mannose by a common phosphoenolpyruvate-dependent phosphotransferase system in Streptococcus mutans GS5. Infect Immun 1984, 43(3):1106.

32. Asanuma N, Yoshii T, Hino T: Molecular characteristics of phosphoenolpyruvate: mannose phosphotransferase system in Streptococcus bovis. Curr Microbiol 2004, 49(1):4.

33. Yebra MJ, Monedero V, Zúñiga M, Deutscher J, Pérez-Martínez G: Molecular analysis of the glucose-specific phosphoenolpyruvate: sugar phosphotransferase system from Lactobacillus casei and its links with the control of sugar metabolism. Microbiology 2006, 152(Pt 1):95.

34. Zúñiga M, Comas I, Linaje R, Monedero V, Yebra MJ, Esteban CD, Deutscher J, Pérez-Martínez G, González-Candelas F: Horizontal gene transfer in the molecular evolution of mannose PTS transporters. Mol Biol Evol 2005, 22(8):1673.

35. Veyrat A, Monedero V, Pérez-Martínez G: Glucose transport by the phosphoenolpyruvate:mannose phosphotransferase system in Lactobacillus casei ATCC 393 and its role in carbon catabolite repression. Microbiology 1994, 140(Pt 5):1141.

36. Francl AL: Functional genomics of carbohydrate utilization in Lactobacillus gasseri. MS thesis University of Illinois at Urbana-Champaign, Food Science and Human Nutrition Department 2008.

37. Lai $X$, Davis FC, Hespell RB, Ingram LO: Cloning of cellobiose phosphoenolpyruvate-dependent phosphotransferase genes: functional expression in recombinant Escherichia coli and identification of a putative binding region for disaccharides. Appl Environ Microbiol 1997, 63(2):355.

38. Old LA, Lowes S, Russell RRB: Genomic variation in Streptococcus mutans: deletions affecting the multiple pathways of $\beta$-glucoside metabolism. Oral Microbiol Immunol 2006, 21(1):21.

39. Cote CK, Cvitkovitch D, Bleiweis AS, Honeyman AL: A novel betaglucoside-specific PTS locus from Streptococcus mutans that is not inhibited by glucose. Microbiology 2000, 146(Pt 7):1555.

40. National Center for Biotechnology Information. http://www.ncbinlm.nih. gov/.

41. Marchler-Bauer A, Bryant SH: CD-search: protein domain annotations on the fly. Nucleic Acids Res 2004, 32:W327.
42. Barrangou R, Altermann E, Hutkins R, Cano R, Klaenhammer TR: Functional and comparative genomic analyses of an operon involved in fructooligosaccharide utilization by Lactobacillus acidophilus. Proc Natl Acad Sci USA 2003, 100(15):8957.

43. Luchansky JB, Tennant MC, Klaenhammer TR: Molecular cloning and deoxyribonucleic acid polymorphisms in Lactobacillus acidophilus and Lactobacillus gasseri. J Dairy Sci 1991, 74(10):3293.

44. Russell WM, Klaenhammer TR: Efficient system for directed integration into the Lactobacillus acidophilus and Lactobacillus gasseri chromosomes via homologous recombination. Appl Environ Microbiol 2001, 67(9):4361.

45. Barboza M, Sela DA, Pirim C, LoCascio RG, Freeman SL, German JB, Mills DA, Lebrilla CB: Glycoprofiling bifidobacterial consumption of galactooligosaccharides by mass spectrometry reveals strain-specific, preferential consumption of glycans. Appl Environ Microbiol 2009, 75(23):7319.

46. Marco ML, Bongers RS, de Vos WM, Kleerebezem M: Spatial and temporal expression of Lactobacillus plantarum genes in the gastrointestinal tracts of mice. Appl Environ Microbiol 2007, 73(1):124.

47. ABI PRISM: Sequence detection system 7700 user bulletin. 2001.

doi:10.1186/1471-2180-10-77

Cite this article as: Francl et al:: The PTS transporters of Lactobacillus gasseri ATCC 33323. BMC Microbiology 2010 10:77.

\section{Submit your next manuscript to BioMed Central and take full advantage of:}

- Convenient online submission

- Thorough peer review

- No space constraints or color figure charges

- Immediate publication on acceptance

- Inclusion in PubMed, CAS, Scopus and Google Scholar

- Research which is freely available for redistribution

Submit your manuscript at www.biomedcentral.com/submit
C Biomed Central 